Archives

40 | 2007

Dépendance(s)

\title{
Liberté formelle et dépendances idéologiques en Afrique
}

Comment déconstruire les relations maîtres/esclaves?

\section{Roger Botte}

\section{CpenEdition}

Journals

Édition électronique

URL : http://journals.openedition.org/ccrh/3396

DOI : $10.4000 /$ ccrh.3396

ISSN : 1760-7906

Éditeur

Centre de recherches historiques - EHESS

Édition imprimée

Date de publication : 25 avril 2007

Pagination : 163-183

ISSN : 0990-9141

Référence électronique

Roger Botte, «Liberté formelle et dépendances idéologiques en Afrique », Les Cahiers du Centre de Recherches Historiques [En ligne], 40 | 2007, mis en ligne le 12 octobre 2011, consulté le 20 avril 2019 URL : http://journals.openedition.org/ccrh/3396; DOI : 10.4000/ccrh.3396

Ce document a été généré automatiquement le 20 avril 2019

Article L.111-1 du Code de la propriété intellectuelle. 


\title{
Liberté formelle et dépendances idéologiques en Afrique
}

\author{
Comment déconstruire les relations maîtres/esclaves?
}

\section{Roger Botte}

1 S'agissant du continent africain, je voudrais rappeler pour mémoire deux débats sur la dépendance auxquels ont contribué les africanistes.

2 Le premier concerne les rapports de l'Afrique et de l'Europe envisagés du point de vue de la dépendance comme théorie du sous-développement. L'argumentaire est bien connu : la rencontre des deux ensembles, l'Europe et l'Afrique, un centre et une périphérie, se serait traduite par la mise en dépendance de l'un par l'autre et aurait entraîné une crise, celle du plus vulnérable des deux partenaires, l'Afrique, dont le continent ne serait toujours pas sorti. La traite négrière transatlantique aurait joué un rôle majeur dans la radicalisation de la subordination. L'économiste Samir Amin est le représentant le plus incisif de cette analyse, également illustrée par les historiens de l'école de Dakar. Aujourd'hui, l'appréciation du phénomène - hors colonisation - apparaît singulièrement plus nuancée, notamment parce que la théorie de la dépendance a l'inconvénient de faire des Africains des sujets passifs de l'histoire'.

3 L'autre débat, je l'évoque à peine, a trait aux relations de dépendance personnelle qui ont trouvé une certaine actualité dans la recherche anthropologique, autour des années soixante-dix, en référence aux travaux des médiévistes. Cette démarche analogique s'est traduite par l'emprunt d'un vocabulaire et de notions telles que celles de fief, de serf ou de suzerain. Jacques Macquet, avec ses travaux sur le Rwanda, en est le représentant le plus connu. Il reste que, pendant un temps, comparer les sociétés interlacustres de l'Afrique orientale et quelques autres à la féodalité occidentale a été un lieu commun propre à l'anthropologie africaniste ${ }^{2}$.

4 Avant d'aborder la question proprement dite de l'esclavage, il faut également esquisser les relations aînés/cadets qui, dans de très nombreuses sociétés africaines, définissent formellement les conditions de reproduction de la hiérarchie sociale et plus particulièrement les rapports de dépendance. Là, le système de classe d'âge, instrument de stratification contrôlé par des "aînés sociaux", se combine en permanence avec 
l'idéologie lignagère pour produire et codifier un système articulant l'appartenance à un groupe et la dépendance personnelle. Dans ce système "emboîté » chacun fournit d'autant moins de travail et en reçoit d'autant plus qu'il se situe plus haut dans la hiérarchie familiale. Je voudrais seulement souligner la différence entre les aînés/cadets libres, d'une part, et les esclaves, d'autre part, et insister sur l'idée que nous n'avons pas affaire à un système en trois groupes (aînés, cadets, esclaves) au sein duquel des aînés exploiteraient, en même temps et de la même façon, les cadets et les esclaves.

La condition des uns et des autres se présente de manière radicalement différente. D'une part, le rapport du maître à l'esclave est un rapport de nature exclusivement économique, résultant d'un achat, d'un héritage ou d'un don, qui donne à ce maître la pleine propriété de la personne de l'esclave - et de sa descendance - de sa force de travail et du produit de son travail. Or, le rapport de subordination existant au sein du groupe des hommes libres soumet le cadet à un tout autre ordre de déterminations : il s'agit entre l'aîné et lui d'un rapport social, certes de domination, mais résultant de la parenté (l'ascendance et la séniorité) et conférant à l'aîné des compétences de gestion et de redistribution des biens. Dans ce cas, la dépendance revêt normalement un aspect transitoire puisqu'en principe les cadets deviennent à leur tour des aînés; en principe seulement car, dans la pratique, la réalité du pouvoir et le bénéfice des biens qu'il suppose, reste circonscrit à un nombre restreint de lignages et peu de « cadets » $\mathrm{y}$ ont accès ${ }^{3}$. Par ailleurs, la détention de la terre reste enserrée dans le réseau des rapports claniques ; les esclaves en sont complètement tenus à l'écart et privés, par conséquent, de tout contrôle sur le moyen de production principal ${ }^{4}$.

\section{L'héritage des sociétés esclavagistes précoloniales}

6 Cette distinction précisée, il est intéressant de prendre comme exemple, à propos de l'esclavage, un même espace géopolitique, celui de la Mauritanie, une société non seulement multiethnique mais également tribalisée et hiérarchisée et, depuis peu, pays pétrolier émergent. Le pays regroupe deux ensembles hybrides ou deux grandes composantes "ethnico-raciales» et culturelles: d'une part, les Maures, "AraboBerbères " arabisés et, d'autre part, diverses sociétés dites "négro-africaines »: les Haalpulaa'ren, les Soninkés (ou Sarakolés), les Wolofs et, dans une moindre proportion, les Bambaras ${ }^{5}$. Leur langue et, dans une certaine mesure, leur mode de vie, leur répertoire culturel, leurs référents historiques distinguent ces groupes les uns des autres, mais tous sont les héritiers de sociétés précoloniales fortement inégalitaires, d'un même imaginaire généalogique aussi et, de fait, leur stratification sociale fait apparaître des systèmes d'ordres largement similaires.

7 En particulier, toutes ces sociétés présentent la même tripartition entre hommes libres, groupes socioprofessionnels ou "gens de castes " et esclaves ${ }^{6}$. Les traits essentiels des formes serviles se sont fixés pour certaines d'entre elles (les Soninkés) dès l'empire médiéval de Ghana autour des VIII IIX ${ }^{e}$ siècles ${ }^{7}$ ou, plus tardivement, à partir du XVIesiècle pour les Haalpulaa'ren et du XVII siècle pour les Maures après l'échec d'une tentative d'instauration d'un État théocratique (guerre de Shurbubba). Le tout dans un contexte géopolitique régional qui a façonné, depuis les premiers temps de la traite transsaharienne, des structures économiques, sociales et culturelles qui se sont conditionnées et influencées mutuellement pendant des siècles, laissant une empreinte indélébile dans les mentalités. 
Toutes ces sociétés ont en commun d'avoir connu différents modes d'organisation du travail, hiérarchiques et dépendants : travail servile «maintenu » des affranchis, travail sous forme de prestations des tributaires, dépendance des non-possesseurs de la terre à l'égard des maîtres du sol, etc. Mais l'esclavage y était dominant, généralisé, omniprésent : il avait cessé d'être un régime de travail parmi d'autres et était devenu la base même de l'ordre social en raison du poids démographique et économique de la population servile. Ceci explique pourquoi des subordinations et des allégeances sociales héritées de ce passé résistent aujourd'hui encore à l'acte juridique et politique que représente l'abolition de l'esclavage, d'abord imposée de l'extérieur par la colonisation française. En fait, s'il existait dans la plupart des sociétés ouest-africaines des modalités de manumission ou même, dans certaines sociétés lignagères, des voies vers l'assimilation, l'idée abolitionniste elle-même leur était parfaitement étrangère. Or, dans le cas précisément de la Mauritanie - où la part des anciens esclaves, toutes communautés confondues, représente probablement au moins la moitié de la population globale - cette abolition a eu lieu à trois reprises (dont la dernière en 1980) . $^{\text {. }}$

9 Aujourd'hui, si les rapports économiques et sociaux restent toujours marqués par de fortes survivances esclavagistes, dont l'intensité varie d'une région à l'autre, d'une tribu ou d'un groupement à l'autre, d'un individu à l'autre, l'économie de marché joue désormais un rôle moteur dans la société mauritanienne et tous les autres rapports lui sont en principe subordonnés. Il conviendrait de dresser pour chacune des quatre sociétés constitutives de la Mauritanie, de la période coloniale à nos jours, les modalités de la progressive et inégale dissolution des relations de dépendance. Je ne traiterai ici essentiellement que de la société maure. Dans les sociétés dites "négro-africaines », la disparition de ces relations prend d'autres formes, différentes les unes des autres; je me contente de les résumer.

10 Dans le cas de la société wolof - plus massivement représentée au Sénégal qu'en Mauritanie -, la création d'un système religieux confrérique, les mourides, avec sa stratification, son idéologie, sa hiérarchie, son mode de reproduction établissant entre ses membres un modèle de rapports non seulement spirituels mais temporels et socioéconomiques vient, vers la fin du XIXesiècle, se superposer, dans le contexte colonial, sur l'ancienne société . Celle-ci conserve ainsi une certaine constance structurelle, tout en se réorganisant dans ce système religieux inégalitaire.

11 Les individus situés au bas de l'échelle sociale dans le système antérieur, les esclaves et les membres des groupes socioprofessionnels (paysans, artisans, traditionistes ou griots, bouffons) sur lesquels ne s'exerçait plus le pouvoir de leurs anciens maitres ou patrons, trouvent alors refuge dans une nouvelle subordination au fondateur de la confrérie, Ahmadu Bemba (mort en 1927), et à ses principaux disciples. Ils deviennent des talibés ou disciples et ils apportent avec eux l'habitude d'une extrême soumission, la volonté d'offrir à un maître religieux leur force de travail ou de lui remettre les fruits de leur travail (ou une partie du produit de celui-ci) afin de gagner leur salut dans l'au-delà ${ }^{10}$. L'acte de soumission à un cheikh, "corps et âme ", revêt une importance vitale dans le système mouride : il est la condition indispensable, et la condition unique, pour devenir membre de la confrérie. Cet acte de soumission est volontaire: le talibé décide seul de qui il reconnaîtra comme son supérieur parmi les cheikhs. Il n'entre donc ici aucun élément de coercition ce qui, à l'évidence, semble distinguer cette relation de celle antérieure de l'esclave wolof à son maître. 

alliances matrimoniales avec les grandes familles de marabouts (les cheikhs) ou bien deviennent eux-mêmes marabouts - bien qu'ils ne fussent pas particulièrement réputés pour leur piété -, et recouvrent ainsi, au moins partiellement, la position prééminente qu'ils avaient perdue. En effet, dans la société wolof d'avant la colonisation, il existait une distinction essentielle entre les esclaves, selon le statut politique de leurs maîtres: esclaves de la couronne ou esclaves royaux et esclaves des gens du peuple. Les esclaves de la couronne constituaient la force armée permanente et le pouvoir ne pouvait se prendre et se garder sans leur concours ${ }^{11}$. Ils étaient beaucoup plus nombreux que les esclaves des gens du peuple. Au moment de la colonisation, ils deviennent les auxiliaires de l'administration coloniale et bénéficient dès lors de commandements et d'avantages considérables: ils exercent le pouvoir au nom des Français, comme ils le faisaient auparavant en tant qu'esclaves de la couronne ${ }^{12}$.

Dès ses origines la confrérie mouride s'organise économiquement autour de la culture de l'arachide. Certes, aujourd'hui, la filière arachidière est en perte de vitesse mais la confrérie contrôle désormais, au sein de filières internationales souvent illicites ${ }^{13}$, d'autres secteurs comme l'immobilier, le marché du médi-cament, le commerce des denrées alimentaires, l'importation de marchandises par container ou l'électronique. La puissance des cheikhs est ainsi considérable au sein de l'économie de marché, dans le système administratif (contrôle des fonctionnaires) et dans l'arène politique, grâce à leur influence sur le vote des talibés. la crise de l'économie marchande (chute des cours de la gomme dans les escales du fleuve Sénégal) et, à la suite de l'abolition française, un début de désagrégation de l'esclavage domestique, engendrent une conjoncture menaçante pour la richesse et le pouvoir des aînés et des notables. Ceux-ci vont alors rapidement se rallier aux desiderata du pouvoir colonial et se faire les médiateurs, à partir de la seconde moitié du XIX ${ }^{e}$ siècle, de l'émigration de leurs dépendants vers le bassin arachidier en pleine expansion ${ }^{14}$.

Dans le cas wolof, la migration constitue une solution propice à une émancipation radicale dans la mesure où la libération de l'esclave ne devient effective qu'à la séparation d'avec le maître. Dans les sociétés aristocratiques et guerrières, comme les Haalpulaa'ren ou aristocratiques et marchandes, comme les Soninkés, la suppression de l'esclavage, c'est-à-dire la perte de leurs bases de reproduction sociale et matérielle, les conduit très tôt à s'investir dans les migrations, alors qu'ailleurs les paysanneries lignagères n'y ont eu recours que beaucoup plus tardivement. Mais, d'une manière générale, chez les Haalpulaar'en comme chez les Soninkés les esclaves dépendent toujours de leurs anciens maitres pour l'accès à la terre, alors que dans la société wolof la position des anciens esclaves ne se différencie plus de celle des paysans libres sans terre.

16 Aujourd'hui, surtout chez les Soninkés, les chefs de famille détenteurs des solidarités familiales nécessaires aux migrants - dont font partie en grand nombre les anciens esclaves - ont réussi à préserver une domination relative sur l'organisation communautaire du groupe et donc à contrôler l'émigration de main-d'œuvre, vers la France notamment ${ }^{15}$. Sur place, en Mauritanie (mais aussi au Mali et, dans une moindre mesure, au Sénégal), la détention des droits fonciers par quelques familles et la délégation de ces droits en contrepartie d'un travail toujours servile constitue un moyen de pression permanent: c'est d'ailleurs une des principales sources du maintien de l'antagonisme « libres/esclaves » au sein même des migrants en France ${ }^{16}$. 

d'émancipation liées aux nouvelles formes d'accumulation monétaire dans le cadre de la migration, la quête de revanche sociale par l'inversion des rôles se heurte encore, lors des cérémonies (mariages, baptêmes, décès), aux impératifs de la relation inférieur/ supérieur. Ainsi, lors du retour périodique d'un migrant «noble» au village, diverses manifestations unilatérales réactivent les marqueurs sociaux : l'« esclave » est tenu de lui apporter un fagot de bois, symbole de comportement servile et la femme de catégorie servile doit lui fournir une bassine d'eau. En outre, l'épouse du migrant «noble » peut demander à une femme d'origine servile de l'«accompagner » dans l'accueil qu'elle réserve à son mari en accomplissant, en son nom, toutes les tâches domestiques pendant la durée du séjour du mari. Ce geste de mise à disposition, d'identification à la servilité, particulièrement fréquent, est évidemment sans réciprocité.

En revanche, pour le migrant « esclave » de retour au village, il est normal de faire un certain nombre de gestes financiers à l'égard de son « ancien maître ». Les cadeaux aux «nobles» d'" esclaves» de retour de la migration sont ainsi fréquents. Ces cadeaux apparaissent d'ailleurs singulièrement ambivalents car s'ils rappellent le fait que les biens de l'esclave appartenaient hier à son maittre, ils manifestent aussi la mise en scène du pouvoir économique de l'« esclave » émigré17.

\section{La société maure entre " hégémonie culturelle » et abolition}

19 La société maure, quant à elle, résiste avec opiniâtreté à l'acte juridique et politique que représente l'abolition de l'esclavage. À l'évidence, l'adoption de mesures légales ne suffit pas à dissoudrepleinement des statuts inscrits dans un réseau complexe de rapports économiques, idéologiques, symboliques.

Certes, la sécheresse qui a frappé le Sahel de 1969 à 1974, son ampleur et sa brutalité, ont détruit les bases écologiques et économiques sur lesquelles reposait, en Mauritanie, l'édifice social traditionnel (élevage, nomadisme et phényciculture); certes, la sédentarisation des nomades qui s'en est suivie a radicalement accéléré, pour un grand nombre d'esclaves (bergers, agriculteurs ou planteurs), le processus de séparation de leurs anciens maîtres en provoquant leur exode massif vers les villes; mais, alors que les anciens maîtres, en tant que groupe social, contrôlent toujours l'ensemble des réseaux économiques et politiques du pays, la masse des anciens esclaves (les haratines) ${ }^{18}$, concentrée dans les bidonvilles, se présente dans des conditions extrêmement défavorables sur le marché du travail ${ }^{19}$.

21 Appartenant aux couches populaires les plus déshéritées, contraints pour survivre de vendre à n'importe quelle condition leur force de travail, ces esclaves, pourtant enfin émancipés, se retrouvent presque autant dominés qu'auparavant et voués à des formes de sujétion à peine différentes de leur condition antérieure ${ }^{20}$. Ils fournissent le contingent le plus important de main-d'œuvre sous-payée pour les travaux les moins valorisés (manœuvres, manutentionnaires, dockers, chauffeurs de taxi, maçons, bouchers, vendeurs d'eau, vendeuses de couscous, etc.) au point que ces emplois ont tendance à se confondre avec la condition haratine; ils sont également les seuls à servir comme domestiques (veilleurs de nuit, gardiens, boys, hommes ou femmes à tout faire), bien 
souvent dans une relation de dépendance non rémunérée au bénéfice des anciens maîtres.

Aussi, en milieu urbain, dans un contexte de rareté et d'insécurité du marché du travail, la recommandation d'une personne plus ou moins influente comme un ancien maître pour un emploi subalterne dans l'administration ou dans une quelconque entreprise, ne se refuse pas ; il existe ainsi des haratines (chauffeurs, plantons...) qui reversent à leur " ancien maître », en une sorte de rétribution de la protection, une part du salaire que leur verse l'administration.

Car, au fond, ni la sédentarisation, ni l'urbanisation, ni l'accès au travail salarié, officiel ou informel, ni l'intégration progressive de valeurs politiques et égalitaires, ni la scolarisation, ni même la globalisation de l'économie de marché ne semblent pouvoir éradiquer définitivement ces formes complexes de subordination précapitalistes qui se révèlent, au contraire, parfaitement compatibles avec des formes modernisées de dépendance.

24 Certes, des individus et même des groupes entiers se sont autonomisés de leurs maîtres mais la règle générale reste cependant la reproduction sous d'autres formes - en particulier sous celle des rapports de dépendance et de clientèle -, des liens serviles préexistants à la sédentarisation en ville.

La nature et les modalités de la sortie de l'oppression esclavagiste constituent un enjeu majeur pour résoudre la concurrence entre la permanence d'ordres sociaux et de loyautés antérieurs et l'institutionnalisation d'un État où, théoriquement, selon la Constitution, tous les citoyens sont égaux en droit. Dans un pays, majoritairement nomade, où jusqu'à l'époque coloniale aucune autorité ne s'était appliquée sur l'ensemble du territoire, la principale difficulté tient au fait que la citoyenneté ne s'incarne pas prioritairement dans les valeurs de l'État mais obéit d'abord à des allégeances situées soit au-delà de l'État, la shari'â, soit en-deçà de l'organisation étatique, les solidarités tribales.

La société maure traditionnelle, à l'instar des autres sociétés du pays, se subdivisait en groupes de statut : des guerriers (groupes dominants de la société), des religieux (groupes maraboutiques dépendants des premiers), des «tributaires» (attachés aux deux précédents groupes), des groupes de libres à spécialisation professionnelle (griots, artisans, forgerons), des haratines ou dépendants d'origine non libre mais affranchis, des esclaves stricto sensu enfin; tous ces groupes étant eux-mêmes inscrits au sein de structures politico-généalogiques, les tribus ; puis, à partir du XVIIesiècle, dans une sorte d'organisation politique centralisée, les émirats (au nombre de quatre), nés entre autres des effets du capitalisme marchand européen. Comme le remarque Abdel Wedoud Ould Cheikh:

Malgré l'expérience vécue et parfaitement répertoriée d'une mobilité statutaire à la fois ascendante et descendante: des guerriers qui deviennent «marabouts» ou «tributaires», des «marabouts» ou des «tributaires» qui deviennent "guerriers", des «esclaves" qui se transforment en anciens esclaves ou hrâtîn, donc malgré cette mobilité réelle, la hiérarchie statutaire ordonnée autour de la généalogie et de l'hypergamie féminine tendait à «naturaliser» sa rigidité, autrement dit à accréditer l'immutabilité du système des ordres dont les esclaves constituaient le degré zéro ${ }^{21}$.

27 Ainsi, pendant des siècles, la société mauritanienne, dans l'ensemble de ses communautés, a fonctionné sur la base d'une stratification sociale figée, à l'image du système des ordres : les individus de par leur naissance, appartiennent à des groupes et 
continuent d'être comptés parmi ces derniers, quels que soient les changements qui s'opèrent dans leurs conditions d'existence - autonomie économique, mobilité sociale. L'individu n'est pas perçu anonymement comme citoyen, mais naît et meurt " esclave ", qu'il soit professeur d'université, officier supérieur, administrateur civil, ouvrier spécialisé ou chômeur. Bref, à la différence des sociétés antiques, le système des idées dominantes continue à appeler « esclave » des personnes dont le statut économique ou social a pourtant fondamentalement changé. D'où l'ambivalence constante entre position sociale "moderne » et hiérarchie traditionnelle dans la société maure, et le caractère immuable de la division noble/esclave dans les sociétés « négro-africaines ».

L'engramme de ce système, celui de représentations nées à l'époque de l'esclavage, alors même que des éléments essentiels du système ont cessé d'opérer, se maintient très largement ${ }^{22}$. Urs Peter Ruf, dans sa thèse sur la fin sans fin du processus de dissolution des relations esclavagistes renvoie à une lecture gramscienne de l'«hégémonie culturelle » des groupes dominants ${ }^{23}$. Il montre, au-delà de l'oppression réalisée au moyen de la coercition, l'emprise plus subtile des groupes dominants sur les consciences des groupes dominés : les haratinesambitionnent d'être intégrés au sein de la société dirigée par les maîtres et leur subordination tiendrait à ce qu'ils renonceraient, pour la plupart, à se placer en dehors de la conception du monde de ces maîtres.Urs Peter Ruf met également en évidence l'ambivalence fondamentalement paradoxale de l'identité des haratines qui veulent, à la fois, manifester leur différence d'avec les Maures et s'identifier à leurs valeurs religieuses, à un espace social et symbolique commun, à leur tribu et à son patrimoine foncier.

29 Cette idéologie du "sens commun " joue à plein pour ces exclus de la généalogie : ils aspirent aux liens de la parenté fictive avec les maîtres alors même que les relations de parenté «de plein droit» sont l'instrument majeur du maintien de la structure hiérarchique tribale et le stratagème pour les tenir à distance $d u$ "bloc historique " (marabouts et guerriers). Tout le dilemme des haratines tient en ces choix contradictoires, assimilation ou résistance, avec pour enjeu la manière dont les relations entre un ancien esclave et son maître vont se disjoindre.

S'agissant du rapport social de la dépendance, il convient toutefois d'envisager une configuration plus complexe et d'écarter les présupposés philosophiques binaires, tel celui qui réduirait la problématique à l'alternative entre coercition et consentement, entre prescription sociale et acceptation individuelle ${ }^{24}$. La dépendance d'individus ou de groupes sociaux les uns envers les autres renvoie aux catégories de pensée auxquels ces individus ou ces groupes ont recours pour appréhender le réel, de sorte que ces catégories, dans toutes ces sociétés à ordres, apparaissent comme "naturelles » ou «normales ». Comme le remarque Benjamin Acloque, le problème, s'agissant desharatines , n'est pas de savoir ou d'ignorer la relation de dépendance qu'ils entretiennent avec autrui, mais, pour employer les concepts de Pierre Bourdieu, de la «méconnaître» comme « violence symbolique $»^{25}$.

31 De fait, les haratines intègrent parfaitement les implications des règles de fonctionnement social construites hors d'eux-mêmes. Ils connaissent la nécessité d'accepter cette dépendance mais savent aussi en jouer : dans l'espoir de rentabiliser un rapport traditionnel de subordination, d'anciens esclaves recréent ou maintiennent volontairement des liens de "subordination symbolique », déclinés sous le langage de la parenté, avec d'anciens " maîtres »; parfois même ce lien est créé ex nihilo, «inventé » pour ainsi dire à des fins d'efficacité sociale. 

cœur même de l'État contemporain. Au fond, la question décisive en revient toujours au statut de l'individu: une rédemption est-elle possible? En Mauritanie, où d'anciens esclaves considèrent que même l'affranchissement n'est pas une dimension de la liberté mais, au contraire, une autre dimension de l'esclavage, celui-ci n'aurait pas de fin. De fait, insidieusement, malgré un ensemble de transformations de toutes natures et malgré la loi, ce que l'affranchissement était censé faire disparaître demeure, c'est-à-dire le stigmate originel de l'ancêtre asservi. Même les membres de groupes affranchis depuis des générations ne sont toujours pas considérés comme des libres à part entière. C'est le cas des descendants de très anciens esclaves du temps des Almoravides, au XI siècle, qui ne voient toujours pas leur état antérieur entièrement oblitéré.

Au fond, pour un individu appartenant aux sociétés dont il est question ici, le meilleur moyen de sortir de la dépendance consiste, non pas à tenter de subvertir la catégorie "esclave» dans sa propre société, mais à intégrer un groupe étranger voisin, en s'efforçant au fil des générations de brouiller la piste de ses origines ${ }^{26}$. Cette défection, au sens qu'Albert $\mathrm{O}$. Hirschmann donne à ce terme, vise à introduire une équivoque, une ambiguïté par l'adoption d'un nouveau statut. Ainsi, échapper à la discrimination ne serait possible que dans une société étrangère car, dans la sienne propre, les éléments constitutifs d'un statut ne peuvent être « oubliés » sans remettre en cause simultanément l'ensemble de la structure globale de la société.

Dans des sociétés où - en contradiction flagrante avec l'appel de l'islam à l'égalité entre les hommes - la question des origines apparait comme une préoccupation lancinante ou comme un fantasme conceptuel, dirait Jacques Derrida, la seule identité valorisée se décline en affichant son nom patronymique. La filiation intervient en effet comme premier principe de classement social, et le simple énoncé par les haratines de leur nom (puisé dans un répertoire immuable: M’Barek, Messaoud, Bilal...) renvoie instantanément, en raison de l'absence d'ascendants reconnus, à une stigmatisation. L'impossibilité de s'affranchir du poids du nom servile, sauf évidemment à en changer ${ }^{27}$, est une des raisons pour lesquelles l'émancipation statutaire n'a pas suivi l'émancipation juridique. Le capital patronymique accumulé par des générations de maîtres, en ligne exclusivement agnatique, souligne a contrario la carence généalogique flagrante des esclaves et de leurs descendants. Cette "violence généalogique» permanente entérine et conforte les hiérarchies sociales et rappelle cruellement que les esclaves relevaient (relèvent) d'un système matrilinéaire où les hommes étaient de simples géniteurs dont la progéniture revenait au maître de la mère. La transmission en ligne utérine de l'identité équivaut à une privation de généalogie et remémore constamment le moment fondateur de l'esclavage: la perte des origines. En outre, en terme de droit musulman, l'esclave est rangé dans la même catégorie que les animaux : il suit le même sort; ainsi, la filiation régissant les esclaves ne ressortit pas d'un modèle social humain, mais d'un modèle biologique comparable à la notion de croît appliqué au bétail ${ }^{28}$.

Simultanément, toutes ces sociétés considèrent, en raison du système d'ordres qui les régit, que la «qualité » d'esclave ou d'homme libre fait partie du patrimoine génétique d'un individu. C'est pourquoi la rémanence du stigmate ${ }^{29}$ est fondée sur le préjugé biologique selon lequel l'individu reçoit de ses ascendants les caractères qui le discriminent et qu'il transmet, à son tour, à ses descendants. C'est parce que la construction des identités sociales relève également d'une rhétorique du sang que cette «biologisation » des rapports sociaux, ce jus sanguinis, permet de reproduire les inégalités 
de naissance. D'où, pour les descendants d'esclaves, la difficulté quasi insurmontable à dépasser la mort sociale originelle et à se faire reconnaître comme personne à part entière dans des sociétés où pour être, il faut avoir été.

\section{L'abolition au regard de la shari'â}

Mais le malentendu le plus profond concerne à l'évidence la monopolisation, par les anciens maîtres, de la parole religieuse, leur gestion du capital symbolique, alors qu'hier justement la mise en servitude prétendait tirer sa légitimité de l'islam. Aujourd'hui encore ce qui se perpétue de l'idéologie esclavagiste puise sa légitimité et ses fondements juridiques dans les enseignements de l'islam et contribue à maintenir la distance entre l'ancien maître et l'ancien esclave. En Mauritanie, "République islamique » dès l'indépendance, l'incompatibilité gît dans l'association même des deux termes laïc et religieux, république et islamique, repris dans la Constitution de 1991. C'est pourquoi l'abrogation de l'esclavage en 1980 - preuve d'ailleurs qu'il avait subsisté tout au long de la colonisation française et qu'il s'était perpétué bien au-delà de l'Indépendance - génère une contradiction difficilement surmontable entre égalité républicaine et inégalité structurelle dans la mesure où le droit musulman établit le principe d'une société inégalitaire fondée sur une stratification entre libres et non-libres. Dès lors, est-il «juridiquement possible d'imposer la suppression d'une institution minutieusement réglée par la shari'â $»^{30}$ ?

Le débat sur la shari'â comme référent législatif remonte au lendemain de l'Indépendance; or, l'érection de la shari'â en unique source de droit (mai 1980) ${ }^{31}$, peu avant le décret d'abolition de l'esclavage (juillet 1980), conforte encore la plupart des juges locaux, les cadis, dans une interprétation réactionnaire des prescriptions islamiques. Ils continuent de tenir compte des pratiques esclavagistes ; ils tranchent presque toujours en faveur des maîtres, rejettent comme irrecevable le témoignagedes esclaves "émancipés " et trouvent des subterfuges dans la tradition pour légaliser les confiscations de terre ou la captation d'héritage (l'« ancien maître » hérite les biens de son « ancien esclave » et non les ayants droit de ce dernier).

Un informateur explique :

En général, ces gens ne laissent personne parmi les esclaves avoir une propriété. Si tu t'adresses à la shari'â [pour te plaindre], celle-ci est au profit de ces gens-là, les bidân, parce que le cadi est un bidân et les autorités sont des bidân. Et moi je suis un sûdan [esclave] " ${ }^{32}$. Il ajoute: «Devant le maître, l'esclaven'est qu'un bien. Si l'esclave est ignorant, on prendra comme prétexte la shari'â pour le déposséder ; s'il est éveillé, on dira que c'est un rebelle et qu'il est en train de semer le désordre [...] Il ne faut pas perpétuer sous d'autres formes la pratique esclavagiste, tantôt en prenant le slogan de la religion tantôt celui de la démocratie.

L'autre registre sur lequel se complaît une lecture esclavagiste de la shari'â est celui du mariage et de l'équivalence de statut social des époux (kêfa'â). Cette notion, centrale en droit musulman, se trouve aujourd'hui convoquée en justice par ceux des parents qui veulent contester le mariage de leur fille avec un conjoint d'un statut ou d'une « caste » jugés indignes ou inférieurs ou avec un membre d'une ethnie différente ${ }^{33}$.

Longtemps, dans l'Afrique de l'Ouest musulmane, les maîtres, en promettant le paradis à leurs esclaves comme récompense de leur travail et de leur obéissance, ont imposé leur domination jusque dans les fins dernières de l'homme. Mieux l'esclave servirait son 
maître, mieux il s'assurerait la grâce divine et éviterait les tourments de l'enfer ${ }^{34}$. L'instrumentalisation des formes du religieux a abouti sur une mystique de la résignation qui, pesant durablement sur la conscience des esclaves, visait à désamorcer tensions sociales et possibles révoltes. Ces croyances imprègnent toujours les mentalités et beaucoup d'«anciens esclaves » - tout autant bons musulmans que leurs maitres ${ }^{35}$ doutent encore de la possibilité d'une libération collective (l'abolition de 1980), et ne jugent finalement comme valide que la formule d'affranchissement unilatéral prononcée par le maître devant témoins: "Tu es libre pour Dieu». C'est justement parce que le maître est toujours considéré comme le meilleur intermédiaire avec Dieu qu'ils lui demandent un acte individuel de libération et sont prêts à lui verser une importante somme d'argent ou son équivalent en nature; ce rachatest la seule façon pour eux de se considérer comme légalement émancipés. C'est-à-dire de devenir, islamiquement parlant, des sujets juridiques à part entière.

Lorsque le colonel Ould Haidallah, promulgue, en juillet 1980, l'ordonnance abolissant l'esclavage, la mesure apparaît davantage comme un constat portant sur l'évolution de la société que comme l'amorce d'un processus nouveau d'émancipation. Il s'agit en fait de répondre aux attentes du mouvement abolitionniste El Hor ("L'Homme libre »), "Organisation de libération et d'émancipation des Haratines ", fondé clandestinement en mars $1978^{36}$, et de consacrer ainsi l'entrée des haratines, en tant que force organisée autonome, sur la scène politique nationale.

L'ordonnance d'abolition - l'État se substituant aux maîtres pour affranchir leurs esclaves - prévoyait d'indemniser les maittres de la perte de la main-d'œuvre qui leur était infligée ${ }^{37}$. En somme, une promesse de rachat des esclaves par l'État en compensation de leur libération comme le firent le Qatar, en 1952, et l'Arabie saoudite, en 1962. Mais l'abolition, implicite en droit moderne et conforme à la Constitution, ne dit rien de ses fondements en droit musulman. Car si l'ordonnance indiquait que la compensation interviendrait conformément à la shari'â, elle restait muette sur les fondements religieux du principe même d'abolition.

En réalité, comme nombre d'ulémas consultés excluaient la possibilité pour l'État de se substituer à l'autorité des maîtres, il fut impossible de justifier la dimension religieuse de l'abolition de l'esclavage, d'établir que les esclaves ne l'étaient plus de «par la volonté de Dieu » et que, désormais, ils accédaient à la «société de Dieu». Cette lacune, peut-être volontaire, permit aux partisans de l'esclavage de déclencher une campagne à l'échelle nationale sur l'illégalité de cette "abolition » qui expropriait les musulmans de leurs biens, acquis en toute légalité : il était, par conséquent, licite pour tout bon musulman de ne pas se conformer à cette ordonnance ${ }^{38}$. D'ailleurs, un grand nombre d'esclaves refusèrent eux-mêmes de se considérer comme libres et de quitter leurs maîtres. L'ordonnance en outre ignore le statut des esclaves déjà affranchis, les haratines, comme si ce statut allait de $\operatorname{soi}^{39}$. Ces défaillances rendaient le texte difficilement applicable et, de fait, vingt-six ans plus tard, les décrets d'application relatifs aux modalités de la compensation n'ont toujours pas été pris.

En 1983, cependant, eut lieu un débat entre théologiens sur la décision d'abolition de l'esclavage. Ceux qui approuvèrent la mesure, comme Hamdal Ould Tâh ${ }^{40}$, discutèrent d'abord les critères de légalité religieuse de la mise en esclavage pour conclure que les origines des mises en esclavage, en Mauritanie, étaient globalement illégales et que, dans ce cas, devait s'appliquer la maxime juridique selon laquelle : «Si l'illicite se mêle avec le licite, on doit éviter le tout ». De ce fait, l'abolition pouvait être considérée comme tout à 
fait légale ; d'autant que l'islam, par ailleurs, collabore avec l'autorité quelle qu'elle soit, même si celle-ci ne remplit pas les conditions prévues par la loi islamique ${ }^{41}$.

Cependant, le discours religieux séculaire qui permettait de légitimer et faire perdurer des situations d'assujettissement se trouve aujourd'hui confronté à de nouvelles formes de religiosité : un phénomène inédit a fait son apparition avec l'implication croissante des haratines dans les mouvements missionnaires fondamentalistes internationaux tels que les Tâbligî jamâ'at d'origine pakistanaise. L'adhésion, à ces mouvements religieux, des couches populaires les plus déshéritées, constituées de la masse des esclaves «libérés ", n'est guère fortuite, car l'offre égalitaire et intégrationniste des nouveaux prêcheurs délégitime la parole religieuse officielle au service des maitres ${ }^{42}$.

En outre, le fait que des descendants d'esclaves puissent devenir imams et diriger la prière au sein de ces mouvements - inconcevable ailleurs - ouvre une nouvelle voie d'émancipation sociale. S'agit-il d'une manière de se forger une "citoyenneté enchantée » qui permettrait de rompre avec un statut social dévalorisant ? L'islam radical est-il en train d'offrir une émancipation religieuse là où l'islam des ulémas et l'abolition juridique formelle ont échoué ? Quoi qu'il en soit, la thématique islamiste s'installe dans un milieu social au poids symbolique, politique et démographique considérable; elle permet aux haratines de satisfaire leur quête d'identité et d'affirmation politique, religieuse et sociale ${ }^{43}$.

Ce mouvement égalitaire et intégrationniste vient de loin. Ainsi, dans les années soixantedix, à une époque marquée par une effervescence politique gauchiste, des haratines détournèrent un genre musical chanté à la gloire du Prophète ${ }^{44}$, traditionnellement conclu par une louange au maître, pour lui substituer des paroles révolutionnaires inspirées du Petit Livre rouge. Ces chants, vite diffusés, eurent un immense succès parmi les haratines: à une félicité future dans l'au-delà succédait une libération dans l'imaginaire.

48 Par un bref détour en Algérie, on observe le même type d'argumentaire religieux et abolitionniste : lors des premières élections législatives pluralistes après l'Indépendance (26 décembre 1991) ${ }^{45}$, dans l'oasis de Djanet, pays des Touaregs Ajjer, le scrutin - à la différence des scrutins antérieurs - devait se dérouler en conformité avec les règles du jeu démocratique. Du coup, le poids démographique des descendants d'esclaves, et donc leur influence électorale, devenait un enjeu capital pour les trois partis politiques en présence. Le parti laïc du Rassemblement pour la culture et la démocratie fit campagne sur le thème de la démocratie assimilée à l'égalité. Dans cette oasis où tant les cadres du Front de libération nationale que ceux du Front islamique du salut (d'ailleurs venus du FLN) appartenaient à la catégorie des maîtres détenteurs des pouvoirs, les deux partis optèrent pour une stratégie de mobilisation de l'électorat des anciens esclaves. Les militants du FLN, devenus subitement extrêmement soucieux du sort des descendants d'esclaves, axèrent leur campagne sur le terme « libération ». Ils mirent en garde contre un vote en faveur du FIS, qui ramènerait les anciens esclaves à leur condition antérieure en remettant en cause les mesures d'accompagnement prises lors de la réaffirmation (implicite) de l'abolition de l'esclavage par Houari Boumediene ${ }^{46}$. Le FIS, de son côté, privilégia le créneau abolitionniste: le vote en sa faveur offrirait aux descendants d'esclaves une véritable émancipation du joug de leurs maitres FLN, en leur garantissant leur salut, et donc leur liberté dans l'au-delà ${ }^{47}$. 


\section{Conclusion}

49 représentés au gouvernement par trois ou quatre ministres et secrétaires généraux, et même, durant deux années, par un Premier ministre ${ }^{48}$. En 1997, le chef de l'État, Maaouya Ould Sid'Ahmed Taya s'enorgueillissait d'avoir fait de la Mauritanie le pays où des « esclaves sont devenus ministres $»^{49}$; mais, onze ans auparavant, lorsque Messaoud Ould Boulkheir fut le premier descendant d'esclaves à accéder à cette fonction ${ }^{50}$ dans l'histoire du pays, on se mit à chantonner le refrain suivant : « Je connais Messaoud qui trait le lait des chèvres, mais Messaoud qui dirige et qui gère... [sous-entendu « on n'a jamais vu ça »] $»^{51}$.

En réalité, la promotion de quelques haratines sert uniquement de faire-valoir dans quelques instances de décision et doit être considérée comme dénuée de toute portée ; il s'agit uniquement d'une approche élitiste de la question, sans commune mesure avec la dimension démocratique de l'enjeu en présence. Le parti politique «Action pour le changement », «parti des opprimés », dénoncera d'ailleurs l'ambiguïté entretenue par l'État : celui-ci,

brandit [...] sa prétendue volonté d'intégration des esclaves et des autres couches opprimées [mais] se limite à exposer comme dans une foire, les rares promus de cette catégorie d'exclus ${ }^{52}$.

51 De fait, la présence devenue nettement plus visible des haratines dans l'arène politique ne modifie pas, pour l'essentiel, la situation de sujétion économique et idéologique qui continue à caractériser les relations du groupe avec les anciens maîtres. Car, indépendamment du caractère incertain de la mesure d'abolition quant à ses effets proprement juridiques, les anciens esclaves, on l'a vu, demeurent dans leur ensemble la couche socialement et économiquement la plus paupérisée de la population.

Toutefois, il existe une réelle diversité des conditions serviles et cette hétérogénéité provoque une indéniable division de ce grand groupe marginalisé : le fils d'esclave ou d'affranchi a un état d'esprit, une façon de voir et un habitus social relativement distincts de celui qui a connu l'esclavage stricto sensu au cours de sa vie ; ce dernier présente luimême un parcours et une mentalité différents de celui dont l'émancipation remonte à quelques générations ; ou encore de ceux qui, n’ayant jamais été émancipés de manière officielle, ont rejeté tout assujettissement et s'autoproclament haratines. Toutes ces catégories, rendues encore plus complexes par le genre - les maîtres émancipent plus volontiers un homme qu'une femme -, ont elles-mêmes peu à voir avec des haratines libres depuis plusieurs siècles ou bien encore avec ceux qui aujourd'hui récusent toute origine servile. Quand la plupart des esclaves subissait une réelle exploitation, d'autres possédaient eux-mêmes des esclaves et, économiquement à l'aise, pouvaient parfois dépasser leur maître en richesse. Enfin, nombre de haratines considèrent que réunir sous cette même appellation tout l'éventail des situations serviles revient, d'une part, à euphémiser le terme esclave, et, d'autre part, à dévaloriser le statut des «vrais » haratines, ceux qui ne se pensent pas comme des descendants d'esclaves et qui utilisent diverses ruses pour être comptés autrement ${ }^{53}$. La difficulté principale des mouvements politiques qui tentent de rassembler toutes ces catégories sous l'unique étiquette d'« opprimés » tient sans aucun doute à ces différences de statuts. 

elles sont évidemment compliquées par différentes variables (politiques, économiques, psychologiques, individuelles) dont la plus importante me semble être le poids toujours considérable de la parenté fictive. En effet, la Risâla de Ibn Zayd, un ouvrage classique de droit malékite écrit au xe siècle, reconnaît au maître qui affranchit son esclave un droit de patronat, ou al-walâ, qui rend l'affranchi inaliénable et incessible : les descendants par les mâles de l'affranchi sont liés à perpétuité à l'affranchisseur et à la famille de celui-ci. Ce droit de patronage, qui s'apparente à un lien clientélaire, représente la justification légale et idéologique du statut de haratine. Ce dernier partage, en principe, tous les droits, mais surtout tous les devoirs des hommes de «sa » tribu, dont il est au demeurant exclu de l'organisation généalogique.

Pour les haratines qui, par définition, ne forment pas une tribu, l'al-walâ est le biais d'accès au système de la solidarité «tribale » du maître au détriment d'une solidarité statutaire, - celle des anciens esclaves entre eux. De fait, le droit de patronage rend impossible la naissance d'une solidarité agnatique forte entre tous les groupes de haratines: ils restent fondamentalement divisés entre les différentes solidarités agnatiques de leurs maîtres, puisque chaque tribu maure intègre ses propres haratines.

La difficulté à rompre les chaînes de la parenté fictive avec les Maures est d'autant plus grande que l'État est incapable de faire face à l'extrême précarité dans laquelle se trouve une grande part des citoyens : en 2005, 46,3\% d'entre eux vivaient au-dessous du seuil de pauvreté. Dans ces circonstances, la solidarité tribale ne constitue plus un archaïsme mais le seul recours dans la lutte pour la survie. Plus largement, pour tous, en l'absence de corps intermédiaires, l'accès aux biens étatiques s'effectue grâce à la médiation de la tribu et par le biais des alliances factionnelles en s'appuyant sur les représentants traditionnels ou néo-traditionnels ${ }^{54}$. L'absence pour les haratines d'un espace autonome identitaire, notamment parce que la prégnance des solidarités restreintes empêche l'émergence de classes sociales, explique concrètement leurs difficultés à se « détribaliser » et à échapper à un système de valeurs qui est celui des maîtres. Leur « consentement » à conserver le lien social de la parenté fictive, synonyme d'entraide, a pour implicite contrepartie leur acceptation d'une condition sociale inférieure.

\section{NOTES}

1. Voir Roger Botte, « Les rapports Nord-Sud, la traite négrière et le Fuuta Jaloo à la fin du XVIII siècle ", Annales ESC, 1991, 6, p. 1411-1435.

2. Par exemple, Jacques Lombard, Structures de type "féodal » en Afrique noire. Étude des dynamismes internes et des relations sociales chez les Bariba du Dahomey, Paris/La Haye, Mouton, 1965 ; Jacques Maquet, «Institutionnalisation féodale des relations de dépendance dans quatre cultures interlacustres ", Cahiers d'études africaines, 1969, IX (35), p. 402-414 ( $\mathrm{n}^{\circ}$ spécial sur « Les relations de dépendance personnelle en Afrique noire »).

3. Par ailleurs, l'accès aux femmes libres permet théoriquement aux cadets d'accéder au rôle d'aîné, voie d'accès évidemment rigoureusement fermée aux esclaves.

Les Cahiers du Centre de Recherches Historiques, 40 | 2007 
4. Voir notamment, Eric Pollet et Grace Winter, «L'organisation sociale du travail agricole des Soninke (Dyahunu, Mali) », Cahiers d'études africaines, 32, 1968, p. 509-534.

5. Par commodité, j'emploie le terme vulgarisé par la colonisation et toujours utilisé. Les Maures s'appellent eux-mêmes al-Bidân, c'est-à-dire «les Blancs». On parle également de manière foncièrement idéologique de «Négro-Mauritaniens", lorsqu'il s'agit d'associer "NégroAfricains » et hrâtîn, ou anciens esclaves, dans une opposition mélanisée aux " Arabo-Berbères » dans la compétition pour le contrôle de l'État. Les Haalpulaar'en, « ceux qui parlent le pulaar, sont les anciens Toucouleurs de la littérature coloniale.

6. L'esclavage comme institution constituait un élément fondamental dans l'organisation des systèmes de production africains mais avec une très grande diversité des réalités sociales. Ainsi, dans les sociétés de la zone sahélo-soudanaise, toutes sont loin d'avoir fait à l'esclavage une place identique. Voir Claude Raynaut et Philippe Lavigne Delville, «L'émancipation de la force de travail », in C. Reynaut (éd.), Sahels, Paris, Éd. Karthala, 1997.

7. Le Galam, par exemple, fut dirigé, sans interruption, par un même groupe dynastique de la fin du VIII siècle jusqu'à la conquête coloniale,à la fin du XIX ${ }^{\mathrm{e}}$ siècle; voir Abdoulaye Bathily, Les portes de l'or. Le royaume de Galam (Sénégal) de l'ère musulmane au temps des négriers (VIII'-XVIII' siècles) , Paris, L'Harmattan, 1989.

8. Le 5 juillet 1980, le Comité militaire de salut national (CMSN) déclare l'« abolition définitive de l'esclavage ». Une ordonnance du 9 novembre 1981 confirme cette abolition (voir infra). Les deux autres étant, d'une part, l'abolition formelle de 1905 par la colonisation française et, d'autre part, celle de facto avec la Constitution du 20 mai 1961 qui réaffirme les grands principes inscrits dans la Déclaration universelle des droits de l'homme de 1948. En 1966 et 1969 des circulaires du ministère de la Justice condamneront « la survivance de pratiques esclavagistes incompatibles avec la Constitution de la République islamique de Mauritanie».

9. Pour Abdoulaye Bara Diop (La société wolof: tradition et changement : les systèmes d'inégalité et de domination, Paris, Éd. Karthala, 1981), le mouridisme est un système essentiellement idéologique, qui agit comme instance de médiation entre le système capitaliste, installé par la colonisation, et la société wolof dont il permet la domination et l'exploitation.

10. La littérature sur les mourides est considérable. Voir, par exemple, Donal Cruise O'Brien, The Mourides of Senegal: The Political and Economic Organization of an Islamic Brotherhood, Oxford, Clarendon Press, 1971. La sainteté des cheikhs étant considérée comme héréditaire, la majorité des marabouts est aujourd'hui représentée par leurs descendants.

11. Ils étaient également gouverneurs de province, percepteurs, etc. Ces esclaves commandaient aux hommes libres et possédaient eux-mêmes des esclaves.

12. À propos de l'ancien royaume (soninké) de Jaara au Mali, Mamadou Diawara (L'empire du verbe, l'éloquence du silence : vers une anthropologie du discours dans les groupes dits dominés du Sahel, Cologne, Rüdiger Köppe, 2003), comparant la manière dont les princes et les serviteurs se servent de la même histoire et comment chacun en tire son profit, met en évidence la nature du pouvoir dont disposent les serviteurs royaux sur les classes supérieures. Certes, ils appartiennent à la catégorie servile, «mais ils sont également parmi les fondements premiers du pouvoir sans lesquels ce dernier est inconcevable. La dichotomie dominé versus dominant est donc inopérante ".

13. Roger Botte, «Économies trafiquantes et mondialisation : la voie africaine vers le développement ?", Politique africaine, 2002, 88, p. 131-150. La diaspora mouride est fortement présente en Europe (France, Italie, Espagne...), par exemple sur tous les marchés hebdomadaires.

14. Philippe David, Les Navetanes. Histoire des migrants saisonniers de l'arachide en Sénégambie des origines à nos jours, Dakar/Abidjan, Les Nouvelles éditions africaines, 1980.

15. Ainsi, dès 1974, une enquête révélait que 30 à $40 \%$ de la population masculine étaient concernés par l'émigration vers la France : Francine Kane, et André Lericollais, « L'émigration en pays soninké », Cahiers Orstom, 1975, 12(2), p.177-187. Voir également, Daniel Delaunay, De la 
captivité à l'exil. Histoire et démographie des migrations paysannes dans la Moyenne Vallée du fleuve Sénégal, Paris, Orstom, 1984.

16. Voir Yaya Sy, «L'esclavage chez les Soninkés : du village à Paris », in Roger Botte (éd).L'Ombre portée de l'esclavage. Avatars contemporains de l'oppression sociale, $n^{\circ}$ spécial du Journal des Africanistes, 2000, 70 (1-2), p. 43-69. Les tensions les plus vives se manifestent notamment au sein des caisses de solidarité (issues de la "Caisse ethnique» de la fin des années cinquante) fondées sur le modèle de l'organisation villageoise avec répartition des tâches entre groupes dominés et groupes dominants. On s'oriente désormais, parfois à l'initiative des immigrés anciens esclaves, vers des caisses séparées (anciens esclaves d'un côté, anciens maîtres de l'autre).

17. À propos des migrations des «anciens esclaves» des Touaregs de la région de Bankilaré (Niger) vers Abidjan, Florence Boyer («L'esclavage chez les Touaregs de Bankilaré au miroir des migrations circulaires ", in Roger Botte (éd.), Esclavage moderne ou modernité de l'esclavage?, $\mathrm{n}^{\circ}$ spécial du Cahiers d'études africaines, XLV (3-4) 179/180, 2005, p. 771-803) montre comment, à la manière d'Alice franchissant le miroir, leur situation dessine une espèce d'alternance entre migration et liberté (en ville) et esclavage et soumission (au campement).

18. J'emploie le terme francisé haratines pour l'arabe hrâtîn (sing. hartâni). Autrefois strictement réservé aux esclaves émancipés le terme hrâtîn, pour diverses raisons - trop longues à développer

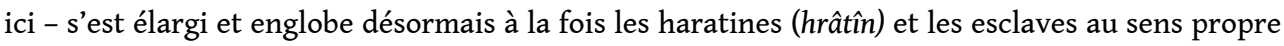
('abîd). C'est dans cette acception large que j'utilise le vocable.

19. Il y a évidemment beaucoup de familles d'anciens maîtres qui, outre leurs esclaves, ont tout perdu ; mais la différence, essentielle, entre ces gens également pauvres tient à leur statut qui contribue toujours à les distinguer.

20. Christiane Rafidinarivo Rakotolahy (Empreinte de servitude, à paraître) constate ceci pour la société malgache: "L'abolition de l'esclavage met à nu le dénuement de l'affranchi. Il n'est propriétaire de rien, héritier de rien si ce n'est d'habitus servile. [...] L'affranchi qui a gagné sa liberté devient la figure emblématique du pauvre.»

21. Abdel Wedoud Ould Cheikh, "Mutations de l'espace public et nouvelles formes de citoyenneté. La difficile sortie des débats sur l'esclavage en Mauritanie », in Zekeria Ould Ahmed Salem ( éd.), Les trajectoires d'un État-frontière, Dakar, Codesria, p. 282-283.

22. Voir Pierre Bonte, "L"'ordre" de la tradition. Évolution des hiérarchies statutaires dans la société maure contemporaine ", Revue du monde musulman et de la Méditerranée, 54 (4), 1989, p. 118-129.

23. Urs Peter Ruf, Ending Slavery. Hierarchy, Dependency and Gender in Central Mauritania, Bielefeld, transcript Verlag, 1998.

24. J'emprunte cette analyse à Benjamin Acloque, «Identité et statut de dépendant en Mauritanie : l'exemple de l'identité sociale des Hrâtîn dans le système segmentaire hiérarchique Bidân ", Nanterre, Université de Paris-X, 1997, mémoire de maîtrise.

25. Pierre Bourdieu avec Loïc J. D. Wacquant, Réponses pour une anthropologie réflexive, Paris, Le Seuil, 1992.

26. Voir Ousmane Kamara, «Les divisions statutaires des descendants d'esclaves au Fuuta Tooro mauritanien », in R. Botte (éd.), L'Ombre portée de l'esclavage [...], op. cit., p. 265-289.

27. Mais le système de double nom ou de changement de nom pose toute une série de questions, notamment dans le cadre de la refonte de l'état-civil en Mauritanie, qu'il est impossible d'aborder ici. Il ne faut pas sous-estimer non plus la volonté d'un certain nombre de descendants d'esclaves de s'approprier, dans un travail d'anamnèse sociale, ce qui stigmatise.

28. «L'esclave nouveau-né est le fruit (ghalla) de sa mère, comme le croît du bétail, et appartient au maître de celle-ci », Mohamed Lemine Ahmed, "L'abolition de l'esclavage en Mauritanie ", Nouakchott, Faculté des sciences juridiques et économiques, 1983, mémoire de maîtrise, p. 33.

29. J'emprunte la notion à Jean-Luc Bonniol, La couleur comme maléfice. Une illustration créole de la généalogie des Blancs et des Noirs, Paris, Albin Michel, 1992, p. 12. 
30. François-Paul Blanc, «L'esclavage à la lumière de la jurisprudence de la Cour suprême de Mauritanie ", in Gérard Chianea et Jean-Luc Chabot, (dir.), Les droits de l'homme et le suffrage universel, 1848-1948-1998, (actes du colloque de Grenoble, avril 1998 ) Paris, L'Harmattan, 2000, p. 355-362.

31. L'organisation judiciaire cependant reste dualiste (droit moderne et droit islamique) tandis que l'application réelle de la shari'â relative aux peines légales (hudud) est considérée, dans les faits, comme inadaptée.

32. Entretien avec Ahmed Ould Hdeîda, habitant au Tagant, Nouakchott, 2001.

33. En Mauritanie où la kêfa'â se situe entre idéologie tribaliste et règle de droit (fiqh) depuis au moins trois siècles, la caution islamique des valeurs tribales prime pour mettre en avant l'importance de la généalogie et de l'endogamie afin d'ancrer la hiérarchie sociale à contre-pied des enseignements religieux, voir Yahya Ould Al-Bara, Fiqh, « Société et pouvoir. Étude des soucis et préoccupations sociopolitiques des théologiens légistes (fuqahas) maures à partir de leurs consultations juridiques (fatawa) $\mathrm{du} \mathrm{XVII}^{\mathrm{e}}$ au $\mathrm{XX}^{\mathrm{e}}$ siècle ", thèse de doctorat en anthropologie, EHESS/Paris, 2001,

34. On transmet dans toutes les tribus maraboutiques, sous forme de parabole, la "révélation » onirique d'un grand saint qui fonde ce principe d'obéissance. Voir Abdalalhi Hormatallah, « Le cri de l'esclave. Pouvoir et esclavage en Mauritanie ", Drôle d'époque, n 12, printemps 2003, p. 79.

35. Le motif religieux peut cependant continuer à légitimer les situations de dépendance : car, en droit, si un musulman libre ne peut être asservi, la conversion à l'islam d'un esclave non musulman n'entraîne pas son affranchissement pour autant.

36. El Hor est directement issu des effets de la sécheresse - exode rural et sédentarisation massive - et des conséquences de la guerre menée contre le Front Polisario au Sahara occidental (1975-1978), les haratines formant le gros des troupes. L'emploi de la majuscule à Haratines, dans nombre de textes, marque le passage du terme à une identité ethnique plutôt que statutaire.

37. Sur les insuffisances de l'ordonnance d'abolition, voir la première analyse critique menée par Mohamed Lemine Ahmed, L'abolition de l'esclavage en Mauritanie, op. cit. (n. 38)

38. Entretien avec El Hassen Ould Benyamine, imam à Nouakchott (bidonville de Teyarett), Al Akhbar, n³7, du 13 au 19 mai 1996. Voir également, Bernard Lewis, Race et esclavage au ProcheOrient, Paris, Gallimard, 1993, p. 117 : «Du point de vue d'un musulman, interdire ce que Dieu permet est un crime presque aussi grand que de permettre ce qu'Il interdit ».

39. Traditionnellement, les Maures reprenaient l'étymologie qui se trouve définie dans le Kitâb al-Istiqsâ: "Libres de seconde catégorie». Le même terme hrâtîn s'applique également aux chevaux et désigne alors un demi-pur-sang, voir Yahya Ould Al-Bara, Fiqh, « Société et pouvoir [...], op. cit., p. 577 (supra note 33)

40. Jurisconsulte renommé, entretien en 2001.

41. Un autre argument pour valider la décision tient à la balance entre deux notions de l'intérêt ( maslaha et mafsada) ou l'opposition entre ce qui est bon ou mauvais ici-bas et au-delà. Maintenir l'esclavage dans un monde qui l'a partout aboli constituerait un grand danger pour la préservation des âmes (amfus). Mieux vaut donc sacrifier les esclaves (en les émancipant) que de perdre son âme.

42. Mais il existe une voie inverse. Ainsi, dans l'Aftout parmi des haratines indépendants depuis longtemps de leurs maîtres, également fortement influencés par les prêcheurs, on assiste à une véritable aspiration à reproduire le modèle religieux dominant. « Ces haratines qui enseignent le Coran à leurs enfants, qui construisent des mosquées, qui fabriquent des tentes de laine comme les Maures traditionnels veulent prendre leur place. [...] Ils veulent réellement devenir des zwaya [catégorie statutaire des religieux] ; ils veulent coûte que coûte acquérir le savoir religieux pour devenir égaux à ceux qui étaient leurs supérieurs ». Entretien avec le poète Ahmedou Ould Abd El Kader, ancien président de la Cour suprême, 2001.

43. Entretien avec le politologue Zekeria Ould Ahmed Salem, 2005. 
44. Ce chant (al-med'h « la Louange »), leur est propre.

45. Entretiens personnels. En février-avril 2002, dans les oasis, à Djanet, In Salah, Goléa, etc., il y eut toute une effervescence pour dénoncer les structures sociales héritées de l'esclavage. Au Touat (localité de Engher), on assista même à des affrontements entre descendants d'esclaves et descendants de maîtres.

46. En 1972, sous le gouvernement de Houari Boumediene, une délégation d'officiels de passage à Djanet réaffirme, lors d'un rassemblement, la conception du citoyen de l'État algérien face à la conception locale et fait afficher sur un panneau devant la mairie le texte suivant : "Aucun être humain ne peut être propriété d'un autre. L'esclavage et le racisme sont strictement interdits en Algérie ".

47. Dans la wilaya d'Illizi dont fait partie Djanet, comme dans toutes les wilayats sahariennes, le FIS n'obtint aucun élu au premier tour. Il n'y eut pas, comme l'on sait, de second tour.

48. Sghaïr Ould M’Barek, juillet 2003-août 2005.

49. Interview au quotidien Chaab du 5 juillet 1997.

50. En tant que ministre du Développement rural, un poste assez conforme à ce que l'on pensait être probablement de la compétence d'un ancien esclave. En 1995, un autre " esclave ", Timera Boubou, issu cette fois de la société soninké, fut également nommé ministre du Développement rural. Il fut rapidement limogé à la suite de très fortes pressions des « libres » de sa communauté, outrés d'être représentés au gouvernement par un "esclave». Le porte-parole des "libres", Sidney Soukhna, était à l'époque chargé des... Droits de l'homme auprès de la Présidence (Abdallahi Hormatallah, "Le cri de l'esclave [...] », p. 84, art. cité supra n. 34)

51. Soit en hassaniyya : na'raf Mes'ûd yahleb fqûd/yaghêr Mes'ûd qâ'id w igûd. La traite, marqueur social, était une activité caractéristique des esclaves et Messaoud un nom typiquement servile. Le terme employé pour «chèvre » (fqûd) souligne encore le statut d'esclave de Messaoud puisqu'il désigne une chèvre qui n'a plus beaucoup de lait (au terme de sa période de lactation) et qu'on laisse généralement à l'esclave. Je remercie Aline Tauzin d'avoir attiré mon attention sur la malice ici du terme « chèvre ». Voir de cette auteure, «Le gigot et l'encrier. Maîtres et esclaves en Mauritanie à travers la littérature orale ", Revue du monde musulman et de la Méditerranée, 51, 1989, p. 74-90.

52. Déclaration de politique générale constitutive (Nouakchott, 17 août 1995). Messaoud Ould Boulhkeir, membre fondateur d'El Hor, est à l'origine de la création de ce parti politique.

53. Voir, par exemple, le cas de Lemine qui, contre toute évidence explique qu'il n'est pas un hartâni mais un khadri (un statut considéré qualitativement différent) dans Meskerem Brhane, « Narratives of the Past, Politics of the Present. Identity, Subordination and the Haratins of Mauritaia " Chicago, University of Chicago, Ill., Ph. D. en sciences politiques, 1997, p. 185.

54. Sous le régime de Maaouya Ould Taya (1984-2005), le clientélisme et le tribalisme ont atteint un niveau critique avec la constitution de groupes oligarchiques aux connivences à la fois économiques, politiques, sécuritaires et tribales. Ainsi des tribus comme les Smacid, les Awled Bou Sba, les Awled Ghaylan ou encore les Idawali et les Rgaïbat comptent dans leurs rangs les plus grands hommes d'affaires du pays. 


\section{AUTEUR}

ROGER BOTTE

EHESS/CEAf 